\title{
Endobronchial Ultrasound in Evaluating Mediastinal Lymphadenopathy
}

\author{
Nandakishore Baikunje ${ }^{1, \odot}$ Giridhar Belur Hosmane ${ }^{1}$ \\ ${ }^{1}$ Department of Pulmonary Medicine, K.S. Hegde Medical Academy, \\ Nitte (Deemed to be) University, Nityananda Nagara, Deralakatte, \\ Mangaluru, Karnataka, India \\ 2Department of Pathology, K.S. Hegde Medical Academy, Nitte \\ (Deemed to be) University, Nityananda Nagara, Deralakatte, \\ Mangaluru, Karnataka, India \\ J Health Allied Sci NU 2021;11:201-203.
}

\author{
Sunil Kumar Y.2
}

Address for correspondence Nandakishore Baikunje, MBBS, MD, DM, Department of Pulmonary Medicine, K.S. Hegde Medical Academy, Nitte (Deemed to be) University, Nityananda Nagara, Deralakatte, Mangaluru 575018, Karnataka, India (e-mail: nandanbaikunje@gmail.com).

\begin{abstract}
Keywords

- tuberculosis

- mediastinal lymphadenopathy

- EBUS-TBNA

Tuberculosis can involve most of the body parts, and tubercular lymphadenitis is common. But isolated involvement of mediastinal lymph nodes without lung parenchymal involvement is rare in adults. A 45-year-old lady presented with subcarinal lymph node enlargement. A conventional transbronchial needle aspiration done at a local hospital was inconclusive. Endobronchial ultrasound-guided transbronchial needle aspiration was done. Cytology showed epithelioid histiocytes and caseous necrosis. Stain for acid-fast bacilli and cartridge-based nucleic acid amplification test for tuberculosis were positive. Endobronchial ultrasound-guided transbronchial needle aspiration is a minimally invasive bronchoscopic technique to visualize and sample mediastinal lymph nodes. However, its availability is limited to specific centers and expertise is required to interpret images and take diagnostic samples.
\end{abstract}

\section{Introduction}

Mediastinal lymphadenopathy is always a diagnostic challenge and can have multiple differential diagnoses. Sampling of mediastinal lymph nodes can be done by either mediastinoscopy, which is a surgical procedure and carries significant risks, or endobronchial ultrasound-transbronchial needle aspiration (EBUS-TBNA), which is a bronchoscopic technique. We present the case of a 45 -year-old female who presented with isolated mediastinal lymphadenopathy without lung parenchymal involvement. EBUS-guided sampling of subcarinal lymph node was done, and the diagnosis of tuberculosis (TB) was achieved.

\section{Case Report}

A 45-year-old female patient from the western coast of India presented with a history of fever for 2 weeks duration along

\section{published online} March 10, 2021
DOI https://doi.org/

$10.1055 / \mathrm{s}-0041-1725241$

ISSN 2582-4287. with dry cough. It was associated with loss of appetite and loss of $5 \mathrm{~kg}$ of weight. There was no history of dyspnea, wheeze, or chest pain. She did not have any history of contact with TB and was not having any preexisting illness. She consulted a local pulmonologist who asked for contrast-enhanced computed tomography of the chest. It showed necrotic subcarinal lymph nodes (-Figs. 1 and 2 ). There were no parenchymal lesions in the lung. A bronchoscopic examination was done, and the endobronchial tree was normal. A conventional TBNA was performed, and smears showed only blood. The patient was referred to our center.

Differential diagnoses of TB, lymphoma, or metastatic malignancy were considered. We performed an EBUS examination and visualized a $3 \times 3 \mathrm{~cm}$ necrotic, heterogenous subcarinal lymph node. EBUS-TBNA was done (-Fig. $\mathbf{3}$ ). A rapid onsite cytologic evaluation was performed, which showed clusters of epithelioid histiocytes against a caseous necrotic background (-Fig. 4). Acid-fast bacilli (AFB) stain
C 2021. Nitte (Deemed to be University).

This is an open access article published by Thieme under the terms of the Creative Commons Attribution-NonDerivative-NonCommercial-License, permitting copying and reproduction so long as the original work is given appropriate credit. Contents may not be used for commercial purposes, or adapted, remixed, transformed or built upon. (https://creativecommons.org/licenses/by-nc-nd/4.0/).

Thieme Medical and Scientific Publishers Pvt. Ltd. A-12, 2nd Floor, Sector 2, Noida-201301 UP, India 


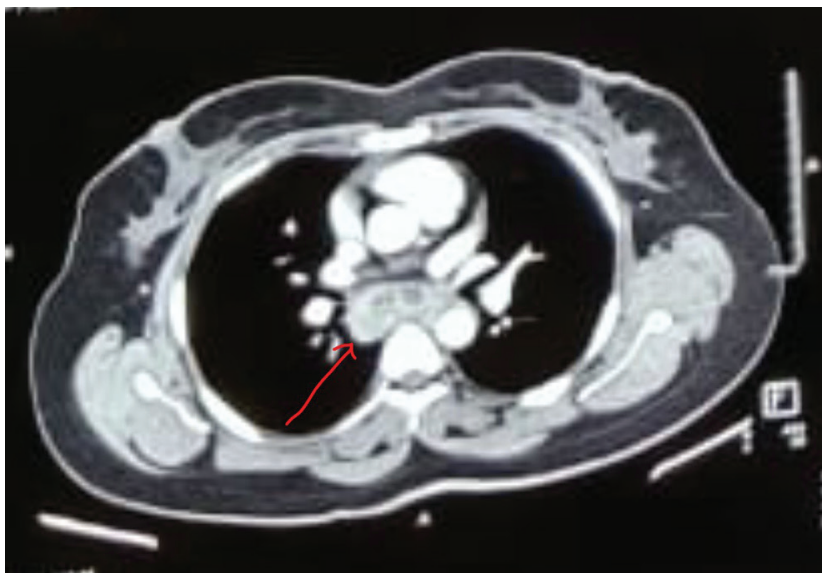

Fig. 1 CECT thorax (transverse view) demonstrating necrotic subcarinal lymph node. Arrow denotes necrotic subcarinal lymph nodes. CECT, contrast-enhanced computed tomography.

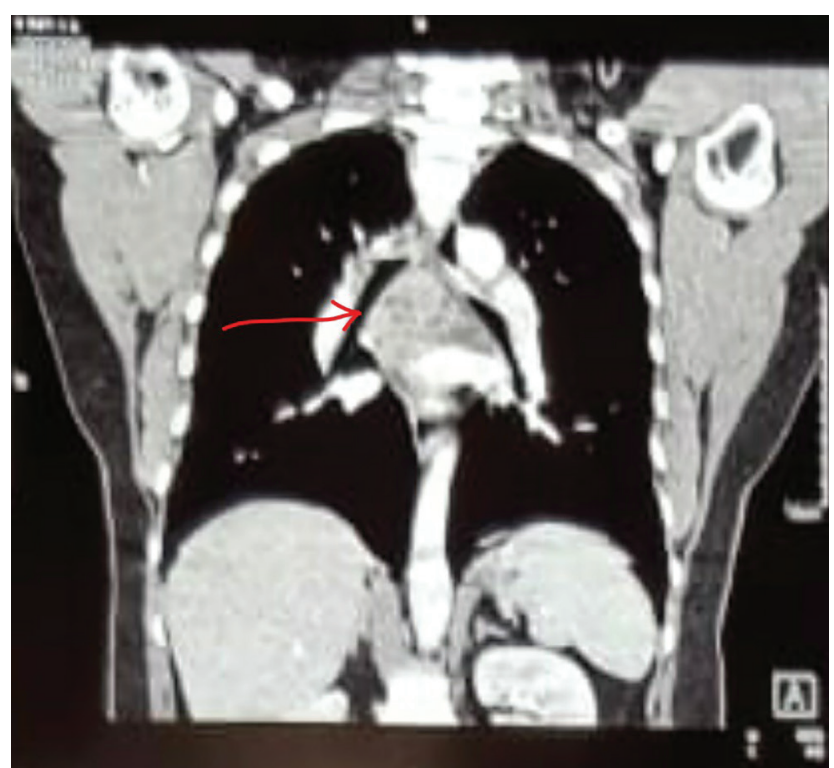

Fig. 2 CECT thorax (coronal view) demonstrating necrotic subcarinal lymph node. Arrow denotes necrotic subcarinal lymph nodes. CECT, contrast-enhanced computed tomography.

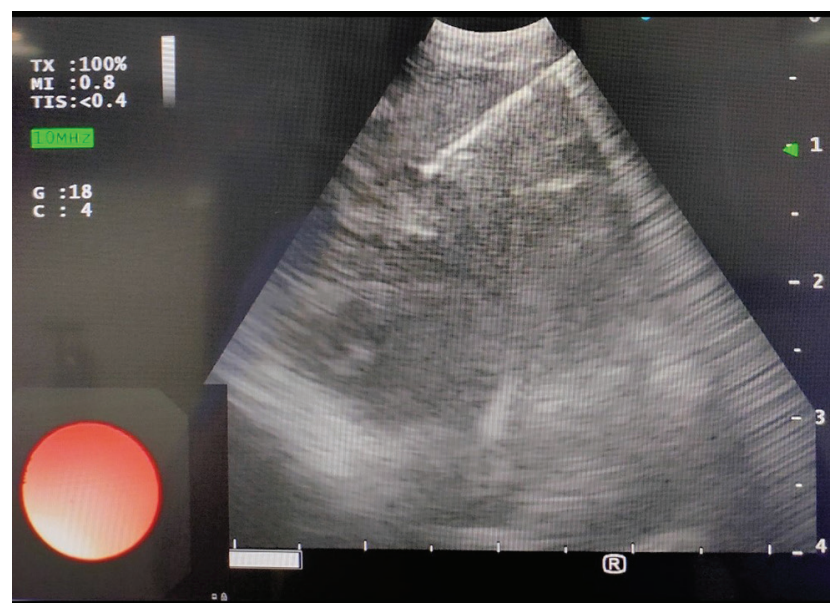

Fig. 3 Endobronchial ultrasound image of necrotic subcarinal lymph node appearing as hypoechoic structure. Transbronchial fine-needle aspiration needle is seen entering the lymph node as a white linear structure in right side of image.

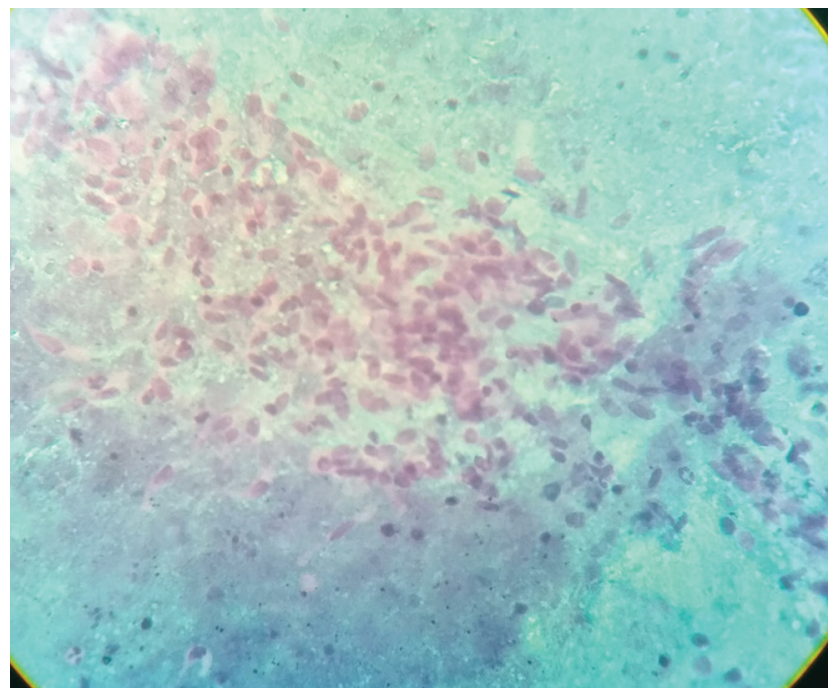

Fig. 4 Granulomas comprising of clusters of epithelioid histiocytes and few lymphocytes against necrotic background (Papanicolaou stain $\mathrm{x} 450 \mathrm{X})$.

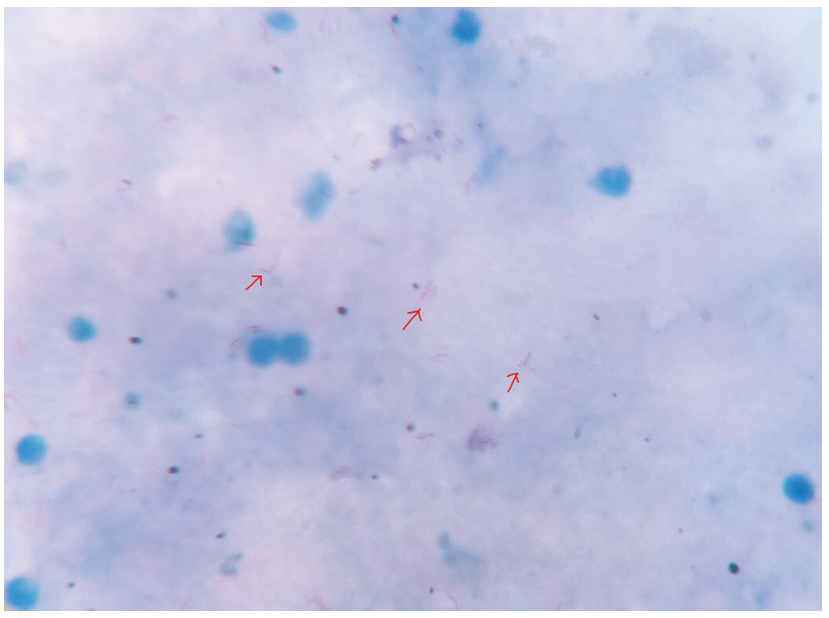

Fig. 5 Ziehl-Neelsen staining showing acid-fast bacilli positivity (arrow).

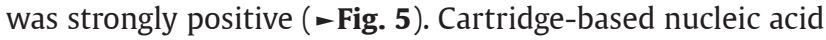
amplification test (CBNAAT) detected Mycobacterium tuberculosis and rifampicin resistance was absent. The patient was started on antitubercular therapy with a directly observed treatment, short-course-based regimen. Her fever subsided in a couple of days and appetite improved.

\section{Discussion}

TB is a highly prevalent infection in developing countries. According to "India TB report 2020," released by India's Ministry of Health and Family Welfare recently, over 2.4 million TB cases were notified in India in 2019. TB can involve most organs of the body and can mimic many other conditions. There is a saying "TB can present like anything except pregnancy." Isolated mediastinal lymphadenopathy is not unusual in children but rarely described in adults in the absence of associated parenchymal lesions. ${ }^{1}$ 
Mediastinal lymph nodes can be enlarged in various pathological conditions like TB, sarcoidosis, lymphoma, and metastatic lung cancer. ${ }^{2}$ A sampling of mediastinal lymph nodes is paramount for diagnosing these conditions and for staging patients with bronchogenic carcinomas. Mediastinoscopy is a surgical procedure for sampling mediastinal lymph nodes but carries significant risks including life-threatening bleeding, and injury to trachea, esophagus, and recurrent laryngeal nerve. ${ }^{3}$

EBUS is a bronchoscopic technique that uses an ultrasound probe at the tip of the bronchoscope to visualize structures within and around airway wall and mediastinum. It is a minimally invasive and safe procedure that can be performed using local anesthesia and conscious sedation. Its availability is institution-specific, and expertise is required to interpret images and obtain diagnostic samples. ${ }^{4}$ Further, EBUS-TBNA is highly accurate with a diagnostic yield of $92 \%$ in diagnosing mediastinal lymphadenopathy. ${ }^{5}$

On ultrasound evaluation with EBUS, heterogenous echotexture of lymph nodes and presence of necrosis favor diagnosis of tubercular lymphadenopathy. ${ }^{6}$ On cytologic examination, both TB and sarcoidosis demonstrate granulomatous inflammation. Presence of caseous necrosis, Ziehl-Neelsen staining demonstrating AFB, detection of Mycobacterium tuberculosis by CBNAAT, or culture clinches the diagnosis of TB. ${ }^{7}$ Besides, CBNAAT identifies mutations that confer rifampicin resistance. ${ }^{8}$ It is important to remember that tubercular lymphadenitis is a paucibacillary disease and AFB positivity may not be seen in nearly half of patients. ${ }^{9}$ In comparison, CBNAAT has better sensitivity and increases diagnostic yield to above $70 \%{ }^{10}$ In remaining cases, the diagnosis will be dependent on cytological findings.

\section{Conclusion}

Tubercular lymphadenitis in mediastinal lymph nodes without parenchymal involvement is rare. EBUS-TBNA is a minimally invasive bronchoscopic procedure that helps in visualizing and sampling mediastinal lymph nodes. Presence of epithelioid granulomas, caseous necrosis, and microbiological tests helps in establishing a diagnosis of TB.

\section{Conflict of Interest}

None declared.

\section{References}

1 Iyengar KB, Kudru CU, Nagiri SK, Rao ACK. Tuberculous mediastinal lymphadenopathy in an adult. BMJ Case Rep 2014;2014:bcr2013200718, doi: 10.1136/bcr-2013-200718

2 Yu C, Xia X, Qin C, Sun X, Zhang Y, Lan X. Is SUVmax helpful in the differential diagnosis of enlarged mediastinal lymph nodes? A pilot Study. Contrast Media Mol Imaging 2018;2018: 341719010.1155/2018/3417190

3 Hammoud ZT, Anderson RC, Meyers BF, et al. The current role of mediastinoscopy in the evaluation of thoracic disease. J Thorac Cardiovasc Surg 1999;118(5):894-899

4 Darwiche K, Özkan F, Wolters C, Eisenmann S. Endobronchial ultrasound (EBUS) - update 2017. Ultraschall Med Stuttg Ger 1980 2018;39(1):14-38

5 Gahlot T, Parakh U, Verma K, Bhalotra B, Jain N. Endobronchial ultrasound-guided transbronchial needle aspiration in diagnosing mediastinal lymphadenopathy. Lung India 2017;34(3): 241-246

6 Erol S, Anar C, Erer OF, Biçmen C, Aydoğdu Z. The contribution of ultrasonographic characteristics of mediastinal lymph nodes on differential diagnosis of tuberculous lymphadenitis from sarcoidosis. Tanaffos 2018;17(4):250-256

7 Gupta N, Muthu V, Agarwal R, Dhooria S. Role of EBUS-TBNA in the diagnosis of tuberculosis and sarcoidosis. J Cytol 2019;36(2):128-130

8 Alemu A, Tadesse M, Seid G, et al. Does Xpert ${ }^{\circledR}$ MTB/RIF assay give rifampicin resistance results without identified mutation? Review of cases from Addis Ababa, Ethiopia. BMC Infect Dis 2020;20(1):87

9 Masilamani S, Arul P, Akshatha C. Correlation of cytomorphological patterns and acid-fast Bacilli positivity in tuberculous lymphadenitis in a rural population of southern India. J Nat Sci Biol Med 2015;6(Suppl 1):S134-S138

10 Dhasmana DJ, Ross C, Bradley CJ, et al. Performance of Xpert MTB/RIF in the diagnosis of tuberculous mediastinal lymphadenopathy by endobronchial ultrasound. Ann Am Thorac Soc 2014;11(3):392-396 